\title{
Noise Enhances Subthreshold Oscillations in Injured Primary Sensory Neurons
}

\author{
Yu-Ying Wang ${ }^{a}$ Zhi-Hong Wen ${ }^{b}$ Jian-Hong Duan ${ }^{a}$ Jun-Ling Zhu ${ }^{d}$ \\ Wen-Ting Wang ${ }^{a}$ Hui Dong ${ }^{c}$ Hui-Ming Li ${ }^{a}$ Guo-Dong Gao ${ }^{d}$ Jun-Ling Xing ${ }^{a}$ \\ San-Jue $\mathrm{Hu}^{\mathrm{a}}$ \\ ${ }^{a}$ Institute of Neuroscience, ${ }^{b}$ Department of Medical Engineering, ${ }^{c}$ Department of Anesthesiology, Xi Jing Hospital, \\ and ${ }^{\mathrm{d}}$ Tang Du Hospital, Fourth Military Medical University, Xi'an, China
}

\section{Key Words \\ Noise $\cdot$ Neurons $\cdot$ Excitability $\cdot$ Resonance $\cdot$ \\ Subthreshold oscillation • Mathematical neuron model}

\begin{abstract}
Noise can play a constructive role in the detection of weak signals in various kinds of peripheral receptors and neurons. What the mechanism underlying the effect of noise is remains unclear. Here, the perforated patch-clamp technique was used on isolated cells from chronic compression of the dorsal root ganglion (DRG) model. Our data provided new insight indicating that, under conditions without external signals, noise can enhance subthreshold oscillations, which was observed in a certain type of neurons with high-frequency $(20-100 \mathrm{~Hz})$ intrinsic resonance from injured DRG neurons. The occurrence of subthreshold oscillation considerably decreased the threshold potential for generating repetitive firing. The above effects of noise can be abolished by blocking the persistent sodium current $\left(I_{\mathrm{Na}, \mathrm{P}}\right)$. Utilizing a mathematical neuron model we further simulated the effect of noise on subthreshold oscillation and firing, and also found that noise can enhance the electrical activity through autonomous stochastic resonance. Accordingly, we propose a new concept of the effects of noise on neural intrinsic activity, which suggests that noise may be an important factor for modulating the excitability of neurons and generation of chronic pain signals.

Copyright $\odot 2011$ S. Karger AG, Basel
\end{abstract}

\section{Introduction}

Since the early 1990s, numerous studies have shown that noise can play a constructive role in the detection of weak signals in various kinds of peripheral receptors $[1-3]$ and neurons [4-6], which suggested that the receptors and neurons can utilize noise to improve their responsiveness. Most of the results were obtained by extracellular recording, which did not reveal the real changes of membrane potential. In a few data using intracellular recording, the changes of spike number induced by noise were shown in hippocampal CA1 neurons $[6,7]$, thalamocortical neurons [8] and pyramidal cells [9]. However, the mechanism underlying effect of noise on membrane excitability is not clear.

In neurons, electrical membrane noise arises from different sources, such as the quasi-random release of neurotransmitters from the synapses and the random switching of ion channels $[4,6,10]$. Recently some experimental and computational studies have reported that channel noise can be a major contributor to electrical membrane noise in neurons, and stochastic behavior of the persistent sodium channel becomes a primary source of channel noise $[4,6,11]$. In addition, nonlinear systems with

Y.-Y. Wang, Z.-H. Wen and J.-H. Duan contributed equally to this work. J.-L. Xing and S.-J. Hu are corresponding authors.

\section{KARGER}

Fax +41613061234

E-Mail karger@karger.ch

www.karger.com
(C) 2011 S. Karger AG, Basel

1424-862X/11/0191-0054\$38.00/0

Accessible online at:

www.karger.com/nsg
San-Jue Hu, Jun-Ling Xing

Institute of Neuroscience

Fourth Military Medical University

Xi'an, Shaanxi 710033 (China)

Tel. +862984774590, E-Mail sjhu@ fmmu.edu.cn; np1403@fmmu.edu.cn 
noise can display stochastic resonance (SR)-like behavior even without external periodic input. This phenomenon has been termed autonomous SR (ASR) $[4,12,13]$. Our previous experimental results have also shown that enhanced persistent sodium current $\left(I_{\mathrm{Na}, \mathrm{P}}\right)$ is one of the major intrinsic factors to determine appearance of both subthreshold oscillation and spontaneous activities in the primary sensory neurons following injury [14-16]. These data suggest that membrane noise may play a certain role during the course of developing hyperexcitability in the injured neurons through enhancing subthreshold oscillation and displaying ASR-like behavior. In order to certify this hypothesis, we selected one type of sensory neurons with subthreshold oscillation from injured dorsal root ganglion (DRG), and examined the effects of electrical membrane noise with a different intensity on both subthreshold oscillations and threshold potential for generating spontaneous activities in an experimental setting and a theoretical model.

\section{Materials and Methods}

\section{Animals and Surgery}

Experiments were conducted on adult Sprague-Dawley rats $(100-250 \mathrm{~g})$ of both sexes in accordance with the National Institutes of Health Guide for the Care and Use of Laboratory Animals (revised in 1996), and were approved by the Animal Use and Protection Committee of our university. The chronic compression of DRG model was prepared according to the method described previously [17]. Briefly, after the conduct of anesthesia with pentobarbital sodium ( $40 \mathrm{mg} / \mathrm{kg}$, i.p.), the L4 and L5 intervertebral foramen on the left side were clearly exposed and a fine, L-shaped stainless steel rod (about $3 \mathrm{~mm}$ in length and 0.4-0.6 $\mathrm{mm}$ in diameter) was inserted into the foramen and left there to produce a steady compression of the DRG. Animals were kept under a natural dark/light cycle, with unlimited access to food and water.

\section{Cell Preparation}

Three to 7 days after surgery, the rats were deeply anesthetized with pentobarbital sodium $(60 \mathrm{mg} / \mathrm{kg}$, i.p.) and L4 and L5 DRGs were removed rapidly. The cells were mechanically dissociated after incubation in culture medium containing trypsin $(0.5 \mathrm{mg} /$ $\mathrm{ml}$; Sigma) and collagenase I ( $1.5 \mathrm{mg} / \mathrm{ml}$; Sigma) for $40-50 \mathrm{~min}$ at $37^{\circ} \mathrm{C}$. Dissociated neurons were plated on coverslips, and then transferred to a recording chamber that was mounted on the stage of an upright microscope (BX50-WI; Olympus Optical, Tokyo, Japan). The chamber was filled with bath solution containing the following (in mM): $150 \mathrm{NaCl}, 5 \mathrm{KCl}, 1 \mathrm{MgCl}_{2}, 3 \mathrm{CaCl}_{2}, 10$ glucose, and 10 HEPES. Recordings were performed on neurons after $1 \mathrm{~h}$. During recording, the neurons were perfused with oxygenated bath solution. Medium-sized cells whose diameters (the average of the widths of its longest and shortest axes) ranged from 30 to $38 \mu \mathrm{m}$ were chosen as objects.

\section{Electrophysiological Recording}

The perforated whole-cell current clamp recording was performed with Axopatch 200 A patch-clamp amplifier (Axon Instruments, USA) at room temperature $\left(\sim 25^{\circ} \mathrm{C}\right)$. The electrode resistance and transient membrane capacitance were compensated by the amplifier. The pipettes were filled with a solution containing (in $\mathrm{mM}$ ): $140 \mathrm{~K}$-glucose, $2 \mathrm{MgCl}_{2}, 3$ EGTA, 10 HEPES and amphotericin B $(100 \mu \mathrm{g} / \mathrm{ml})$. In some experiments, in order to isolate $I_{\mathrm{Na}, \mathrm{P}}$, the pipette solution contained (in $\mathrm{mM}$ ): $140 \mathrm{CsF}$, 1 EGTA, $10 \mathrm{NaCl}, 10$ HEPES, while the extracellular solution contained (in mM): $140 \mathrm{NaCl}, 3 \mathrm{KCl}, 1 \mathrm{MgCl}_{2}, 1 \mathrm{CaCl}_{2}, 10$ HEPES. All solutions were filtered through $0.22 \mu \mathrm{m}$ cellulose acetate filters. In voltage-clamp mode, the persistent sodium current was elicited by a depolarizing ramp from holding potential of -80 to $-30 \mathrm{mV}$. Data acquisition was filtered at $4 \mathrm{kHz}$ and sampled at $10 \mathrm{kHz}$ with the Clampex 9.0 software system (Axon Instruments) through the digitizer (digidata 1322A, Axon Instruments), and the analysis was carried out by Clampfit 9.0 (Axon Instruments) and origin6.1 (OriginLab Corp., USA). The spike threshold is defined as the voltage at which the value of $d V / d t$ preceding a spike first became $\leq 1 / 30$ of $d V / d t_{\max }$ as previously described [18].

\section{Noise Input and Signal-to-Noise Ratio Measuring}

The additive electrical noise is Gaussian white noise which is produced by software Labview in computer, and the noise with different intensity ( $D$, pA r.m.s.) was injected into the recorded DRG neurons through the same recording microelectrode via patch-clamp amplifier. Signal-to-noise ratio $(\mathrm{SNR})=\mathrm{hf} / \Delta \mathrm{f}$, where $h$ and $f$ represent the height and frequency of the main peak in the power spectrum of the output data, respectively. $\Delta \mathrm{f}$ represents the frequency width of the peak at the height of $1 / 2 \mathrm{~h}$. The SNR actually reflects the degree of coherence, i.e. $\beta[12,13]$.

\section{Mathematical Neuron Model}

The neuron model, for observing oscillatory behavior, is an isopotential RC compartment with three discrete transmembrane ionic currents flowing in parallel to the membrane capacitance. The current equation for this model is:

$$
C \frac{d V}{d t}+I_{K, S}+I_{N a, P}+I_{\text {leak }}+I_{i n}=0
$$

$C$, the membrane capacitance, is $0.25 \mathrm{nF}, I_{\mathrm{K}, \mathrm{S}}$ is slow noninactivating potassium current and $I_{\mathrm{Na}, \mathrm{P}}$ is persistent noninactivating sodium current. $I_{\text {leak }}$ is the passive current and $I_{\text {in }}$ is the input current. Two types of current $\left(I_{\mathrm{K}, \mathrm{S}}\right.$ and $\left.I_{\mathrm{Na}, \mathrm{P}}\right)$ are used to simulate the production of subthreshold oscillation. In some simulations, for generating spike, two additional voltage-dependent currents are added to this model, a fast sodium current $\left(I_{\mathrm{Na}}\right)$ and a delayed potassium current $\left(I_{\mathrm{K}}\right)$. The equation is:

$$
C \frac{d V}{d t}+I_{\mathrm{Na}^{+}}+I_{K^{+}}+I_{K, S}+I_{N a, P}+I_{\text {leak }}+I_{i n}=0
$$

Details of the model equation and parameters can be found in the paper by Gutfreund et al. [19]. The mathematical model was solved by Simulink block of Matlab. To examine the subthreshold oscillation and the action potential induced by noise, Gaussian white noise is added to the above models, respectively. All simulation is done at depolarization levels. So,

$$
I_{i n}=D C+D \xi(t)
$$


D is the intensity of Gaussian white noise $\xi(\mathrm{t})$ with zero mean value $\langle\xi(\mathrm{t})\rangle=0$ and unit variance $\left\langle\xi(\mathrm{t}) \xi\left(\mathrm{t}^{\prime}\right)\right\rangle=\delta(\mathrm{t})$. For observing oscillatory and fire behavior, membrane potential was set to near threshold. In simulation, DC is $1.3 \mathrm{nA}$. Noise $\xi(\mathrm{t})$ was generated by Band-Limited White Noise block in Simulink Sources. In block parameter settings, noise power (intensity) is set as D, others are default.

This mathematical model mainly described the properties of resonance for neurons. These neurons exhibit subthreshold oscillations and spikes based on the oscillations. Moreover, $I_{\mathrm{Na}}$, p play an important role in the occurrence of the oscillations. So this model could be close to simulating the basic characteristics of DRG neurons we recorded in the experiments.

\section{Drug Application}

TTX was purchased from Sigma-Aldrich Cookson Ltd. Drugs were applied by changing perfusing solution from bath one to drug one.

\section{Results}

\section{Repetitive Firing from Two Types of Injured DRG}

\section{Neurons}

In 150 neurons examined, two types of neurons with repetitive firing in response to input depolarization ramp currents were observed. The first type, 15 neurons (about $10 \%)$, exhibited tonic repetitive firing with a lower threshold and did not appear to have regular subthreshold oscillations, and also showed lower frequency $(\leq 5 \mathrm{~Hz})$ resonance (fig. 1a-c). The second type, 46 neurons (about $30 \%$ ), always exhibited resonance properties with a higher frequency ranging from about 20 to $100 \mathrm{~Hz}$ (fig. 1d-f). This type of neurons always exhibited regular or spindle subthreshold oscillations and burst firing in response to depolarizing ramp current input, and spikes arose from the subthreshold oscillations. With the membrane potential depolarizing, the number and amplitude of subthreshold oscillations increased, which are similar to those shown previously in the injured primary sensory neurons [20, 21]. Other neurons (about 60\%) did not exhibit repetitive or burst firing, and regular subthreshold oscillations in response to depolarizing ramp currents, while they only generated single or a few spikes to depolarizing step stimulation (data not shown).

\section{Effects of Noise on Repetitive Firings}

For the first type of injured DRG neurons, when noise was added with an increase in intensity from 30 to $250 \mathrm{pA}$ r.m.s. the threshold potential of first spike and numbers of repetitive firings did not exhibit any significant changes $(n=12)$ (fig. $2 a, b)$, in spite of small irregular fluctua- tions appearing on resting membrane potential. When the noise was added with an increase in intensity from 30 to $250 \mathrm{pA}$ r.m.s, the second type of neurons showed that the threshold potential of the first spike changed from -20.9 to $-35.5 \mathrm{mV}$ (fig. $2 \mathrm{c}$ ). Summarized data were shown as in figure 2d; the threshold potential for the first spike decreased from $-17.7 \pm 2.0$ to $-26.8 \pm 2.3 \mathrm{mV}(\mathrm{n}=12)$. In the meantime, the number of spikes increased from 3.2 \pm 0.8 to $13.6 \pm 5.0(\mathrm{n}=12)$ along with the additional increased noise (30-120 pA r.m.s.). We plotted the relationship between SNR and noise intensity in the neurons with subthreshold oscillations as shown in figure 2e. The typical shape of SNR demonstrated that the optimal noise intensity with a maximal presence of SNR occurs, suggesting that an ASR behavior appears in the second type of neurons $[12,13]$.

\section{Effects of Noise on Subthreshold Oscillations}

As shown in figure $3 a$, the membrane potential for initially emerging oscillations in a second-type neuron changed from -24.1 to $-47.6 \mathrm{mV}$ by adding noise with different strengths to the same ramp current. Figure $3 \mathrm{c}$ shows the statistical results of membrane potential for emerging oscillations $(n=9)$. The average amplitudes of subthreshold oscillations increased significantly from 3.0 \pm 0.6 to $7.5 \pm 0.8 \mathrm{mV}(\mathrm{n}=9$, fig. $3 \mathrm{c})$. For neurons with subthreshold oscillations, Fourier analysis revealed that the power of dominant oscillation gradually rose with the increase in noise intensity within a certain range, reflecting a noise-dependent increase in the amplitude and coherence of the oscillations (fig. 3b). However, for neurons without subthreshold oscillation, the first type of neurons, noise did not have a significant effect on the membrane potential (data not shown).

\section{$I_{N a, P}$ Involved in Effect of Noise}

It is known that $I_{\mathrm{Na}}$, p plays an amplifying role in the amplitude of oscillations [6,22]. To determine whether $I_{\mathrm{Na}, \mathrm{P}}$ exists in the DRG neurons with subthreshold oscillation, a ramp voltage command from -80 to $-30 \mathrm{mV}$ was applied. As shown in figure $4 \mathrm{a}$, a current was first activated at about $-70 \mathrm{mV}$ and the amplitude peaks to $\sim 250$ $\mathrm{pA}$ at about $-45 \mathrm{mV}$. This current can be abolished by application of a low dose of TTX (100 nM), which is similar to $I_{\mathrm{Na}, \mathrm{P}}$ in some other sensory neurons [23]. To examine the role of $I_{\mathrm{Na}, \mathrm{P}}$ in the process of noise-induced subthreshold oscillation, we compared the effects before and after blocking of $I_{\mathrm{Na}, \mathrm{P}}$ on noise-induced subthreshold oscillation in 5 neurons. As shown in figure $4 \mathrm{~b}$, at base, a smaller depolarizing ramp current did not cause any ob- 


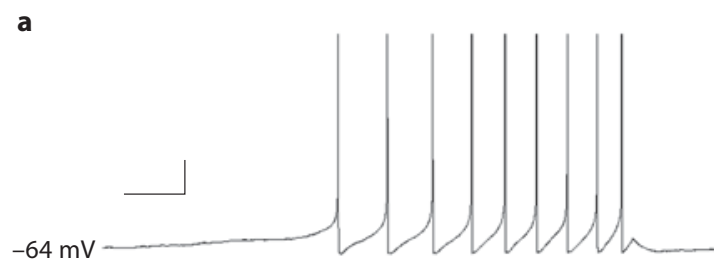

$0.1 \mathrm{nA}$

b

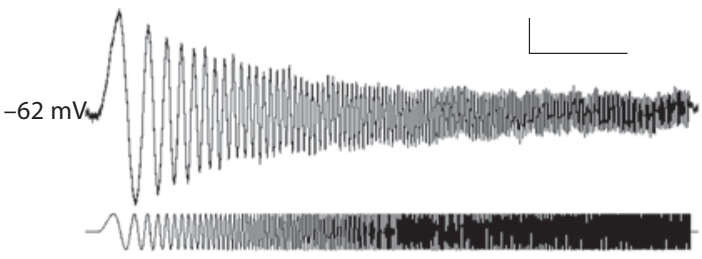

Input ZAP current

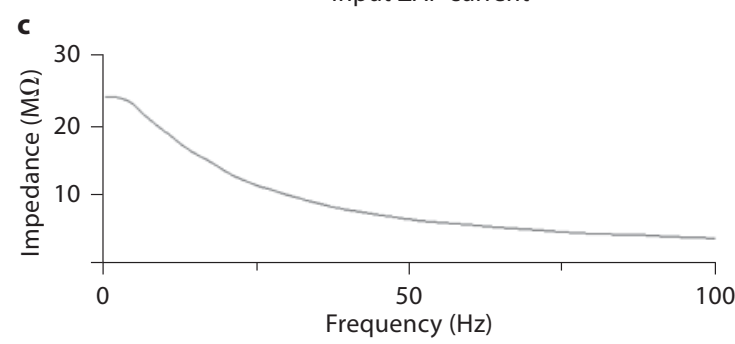

d

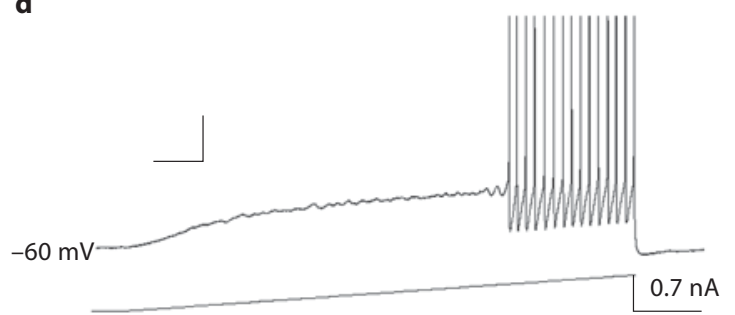

e

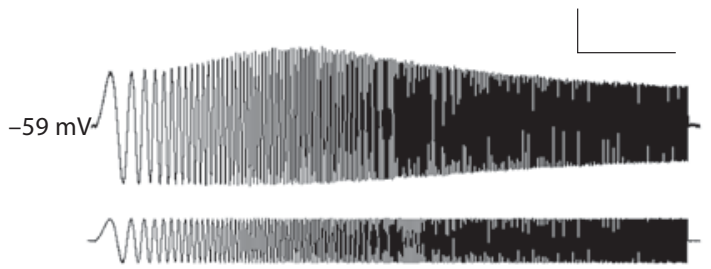

Input ZAP current

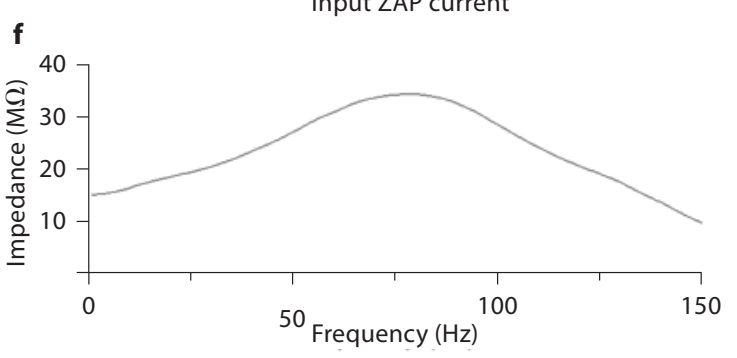

Fig. 1. Subthreshold membrane resonance in injured primary sensory neurons of type $1(\mathbf{a}-\mathbf{c})$ and type $2(\mathbf{d}-\mathbf{f})$. a Repetitive firing was evoked by depolarizing ramp current, which is dependent on current intensity in a type 1 neuron. Scale (y, x) $10 \mathrm{mV}, 100 \mathrm{~ms}$. d Depolarizing ramp current evoked a rhythmical bursting with subthreshold membrane potential oscillations, which is not sensitive to current intensity in a type 2 neuron. Scale (y, x) $20 \mathrm{mV}, 50$ $\mathrm{ms}$. b, e Voltage response (upper trace) of neurons injected with

vious subthreshold oscillations in an injured DRG neuron. While adding noise with a certain intensity under the same conditions, subthreshold oscillations and spikes occurred obviously. Then, the application of a low dose of TTX (100 nM) gradually abolished both subthreshold oscillations and discharges induced by noise. Note that when a discharge series disappeared, the action potential can still be evoked by a short pulse (fig. $4 \mathrm{~b}$, inset), indicating that the ability of neurons to discharge remained. By washing out TTX, which might reverse the effect of $I_{\mathrm{Na}, \mathrm{P}}$, the noise can again evoke the obvious subthreshold oscillations as well as spikes (fig. 4b, upper traces).

Noise Enhances Subthreshold

Oscillations in Injured Neurons
ZAP current (lower trace; from 0 to $100 \mathrm{~Hz}, 3 \mathrm{~s}$ in $\mathbf{b}$ and from 0 to $200 \mathrm{~Hz}, 3 \mathrm{~s}$ in $\mathbf{e}$ ). The type 1 neuron responded at the peak of a very low frequency (b), the type 2 neuron, on the other hand, at a high frequency (e). Scale (y, x) $1 \mathrm{mV}, 500 \mathrm{~ms}$ for $\mathbf{b}$ and $6 \mathrm{mV}, 500$ $\mathrm{ms}$ for e. c, $\mathbf{f}$ The impedance curve plotted as a function of input frequency, using the data in $\mathbf{b}$ and $\mathbf{e}$. It shows that the resonance frequency is about $5 \mathrm{~Hz}$ for type 1 neurons and about $75 \mathrm{~Hz}$ for type 2 neurons. Spikes in $\mathbf{a}$ and $\mathbf{d}$ were truncated.

\section{Mathematical Neuronal Model}

A mathematical neuron model as mentioned above was used to confirm the effects of noise on electrical activities. As shown in figure $5 \mathrm{a}$, by first adjusting the depolarizing step current to keep the neuron model near resting state and not displaying oscillations, and then adding noise with increasing intensity, subthreshold oscillation can be triggered and enhanced. When further increasing noise intensity, both the amplitude of subthreshold oscillation and the number of spikes increased obviously. The plot of the relationship between SNR and noise also shows a characteristic of ASR (fig. 5b). The co- 


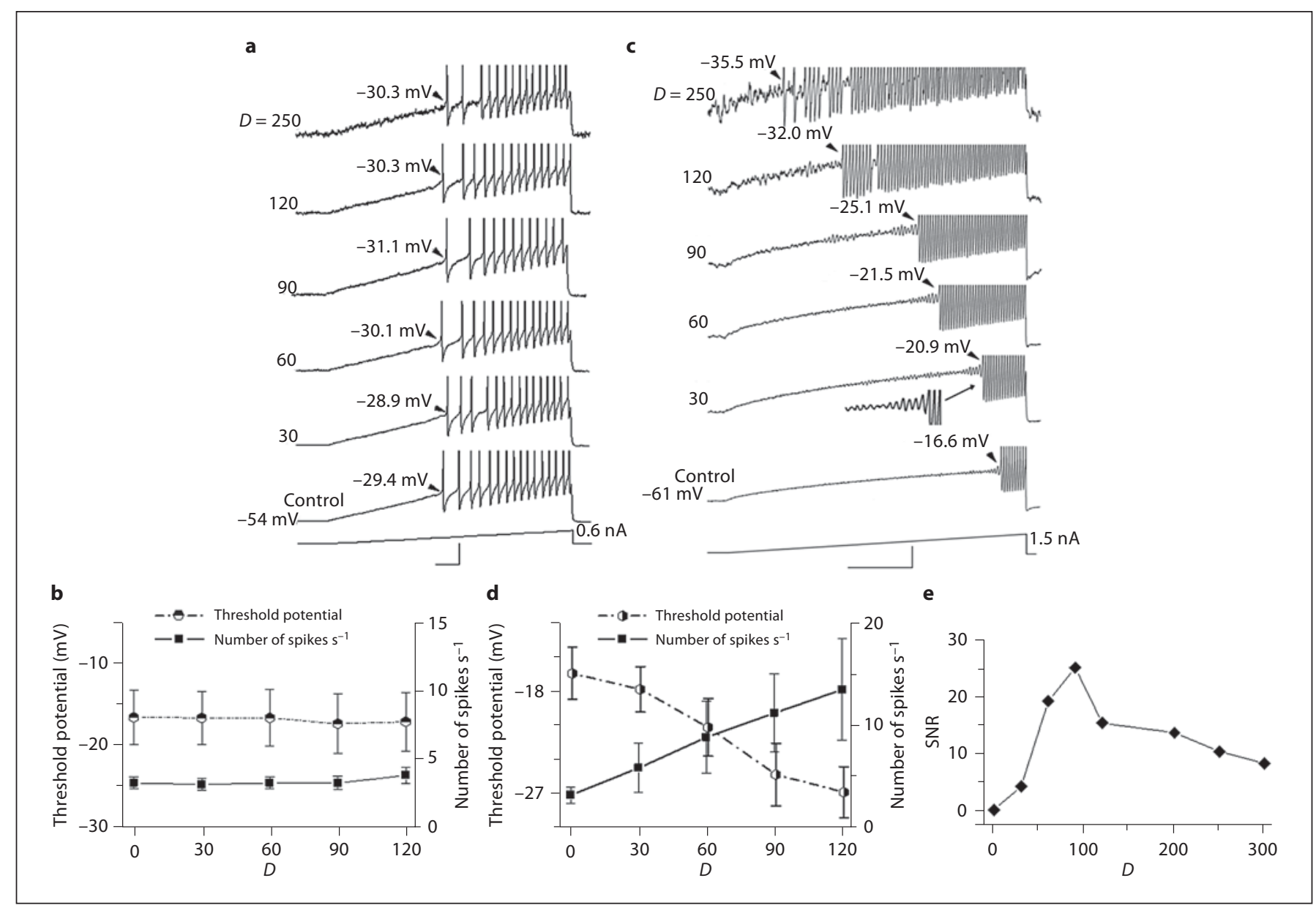

Fig. 2. The effects of noise on spike threshold in injured DRG neurons. a Responses of a type 1 neuron to the injected ramp current with noise of different intensity $(D, \mathrm{pA}$ r.m.s. note on the right of each curve). The arrowheads mark the first spike threshold potential. Scale (y, x) $20 \mathrm{mV}, 100 \mathrm{~ms}$. c Responses of a type 2 neuron to the injected ramp current with noise of a different intensity. Along with the increment of noise intensity, the reduction of first spike threshold potential as marked by arrowheads and the increase of spike numbers were observed, respectively. Scale (y, x)

herence of these noise-induced oscillations is shown to be maximal for a certain noise intensity. Qualitatively, the neuron model correctly predicted the behavior of real sensory neurons induced by noise (see fig. 2e), suggesting that noise-induced oscillation may reflect one kind of universal feature of neurons with intrinsic oscillatory property. This result is basically consistent with some observations that the mathematical neuron models in the presence of noise can also display SR-like behavior even without external signal [13].
$5 \mathrm{mV}, 200 \mathrm{~ms}$. Action potentials shown in a and $\mathbf{c}$ have been truncated. The inset (next to the arrow) in c displayed the obvious subthreshold oscillation. $\mathbf{b}$ The broken line represents the mean threshold potential vs. noise intensity and the solid line represents the mean spike numbers $\mathrm{s}^{-1}$ vs. noise intensity in the neurons of type $1(\mathrm{n}=12)$. $\mathbf{d}$ The notes are the same as in $\mathbf{b}$ in the neurons of type 2 with subthreshold oscillations $(n=12)$. e SNR vs. noise intensity for the same neuron shown in c.

\section{Discussion}

In the present study, a novel phenomenon observed is the great reduction of spike threshold induced by noise, which may be related to the following causes. First, the membrane potential for emerging subthreshold oscillation drops greatly, which is induced by noise as described above. In the injured DRG neurons, the subthreshold oscillation is considered as a fundamental factor for generating ectopic firing [20,21]. Second, due to the augmentation of amplitude of subthreshold oscillation during the 


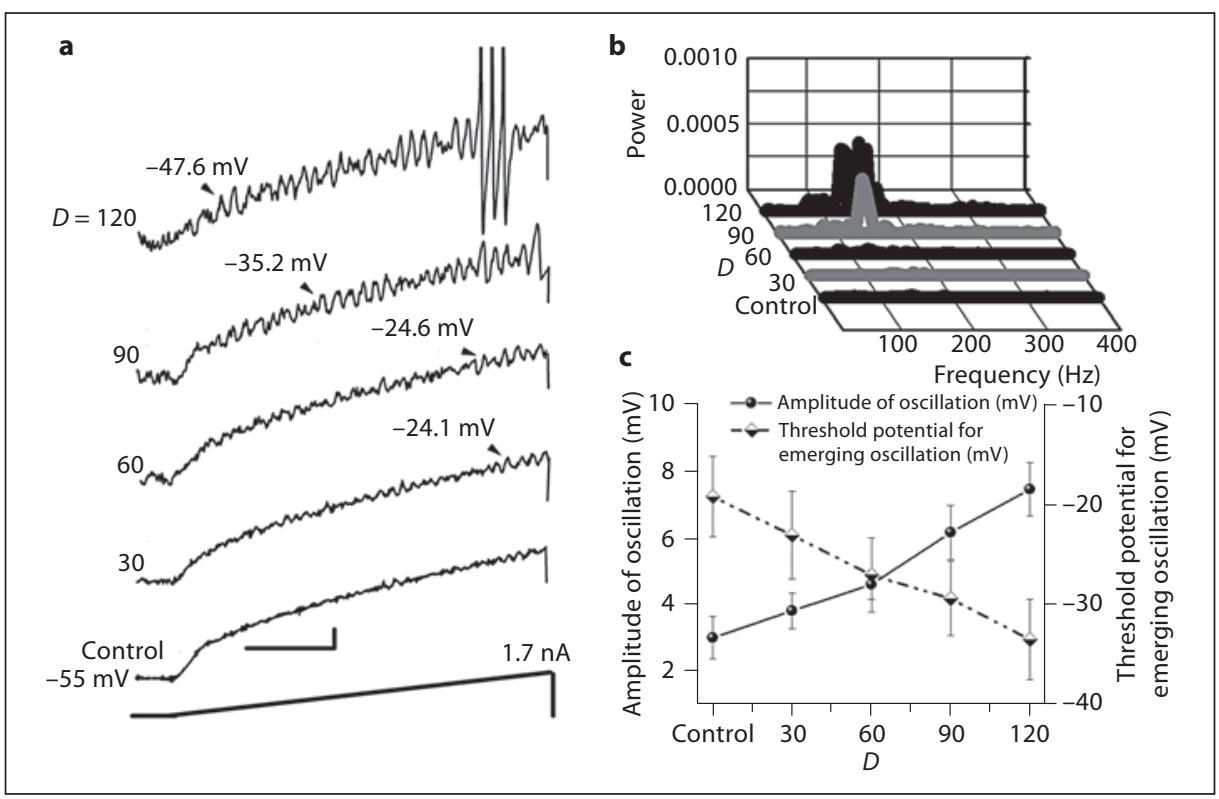

Fig. 3. Noise-enhanced subthreshold oscillation in the injured DRG neurons. a Effect of noise with different intensities $(D, \mathrm{pA}$ r.m.s. note on the left of each curve) on the subthreshold oscillation, and the arrowheads mark the membrane potential for generating subthreshold oscillations. The action potentials have been truncated. Scale (y, x) $5 \mathrm{mV}, 50 \mathrm{~ms}$. b The fast Fourier analysis profile in the cell shown in a illustrates the increase in both oscil- lation coherence and oscillation amplitude (power) with increment of noise intensity. c The broken line represents the mean level of membrane potential for generating subthreshold oscillations vs. noise intensity and the solid line represents the mean amplitude of subthreshold oscillations versus noise intensity $(n=9)$.

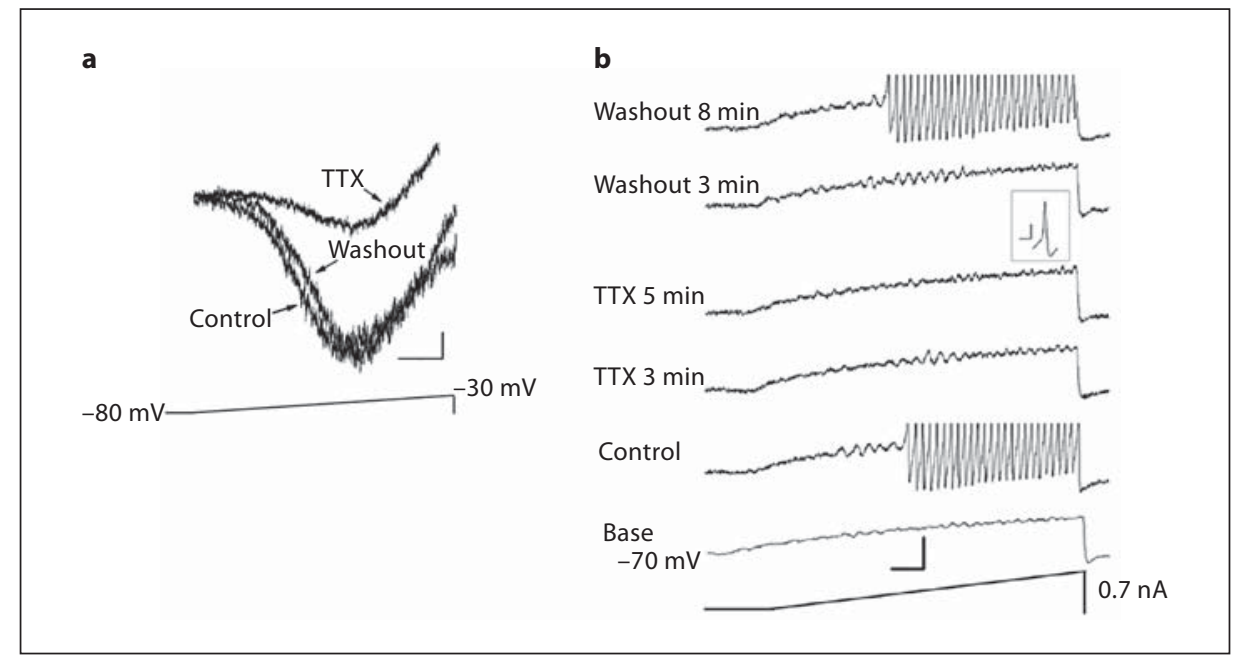

Fig. 4. Noise-induced subthreshold oscillation mediated by persistent sodium current $\left(I_{\mathrm{Na}, \mathrm{P}}\right)$. a Current-voltage curve of $I_{\mathrm{Na}, \mathrm{P}}$ obtained by applying a depolarizing ramp voltage from -80 to -30 $\mathrm{mV}$. The upper panel shows the traces of $I_{\mathrm{Na}, \mathrm{P}}$ at control, application of TTX $(100 \mathrm{nM})$ and after TTX washout. Scale $(\mathrm{y}, \mathrm{x}) 40 \mathrm{pA}$, $200 \mathrm{~ms}$. b Effect of TTX on subthreshold oscillations and spikes which were induced by noise. From bottom to top, the base represents the membrane potential only with an injected ramp current (up to $0.7 \mathrm{nA}$ ); the control represents the effects of an added noise with a fixed intensity (60 pA r.m.s.) on the same ramp current as applied on the base; obvious oscillation and firing were evoked. The upper four traces indicate application of TTX $(100 \mathrm{nM})$ and after TTX washout. Note that after application of TTX for 3-5 min, most of subthreshold oscillations and resulting spikes disappeared while the spike can be evoked by intracellular stimulus (inset, scale (y, x) $20 \mathrm{mV}, 5 \mathrm{~ms}$ ). The action potentials have been truncated. Scale (y, x) $20 \mathrm{mV}, 50 \mathrm{~ms}$. 


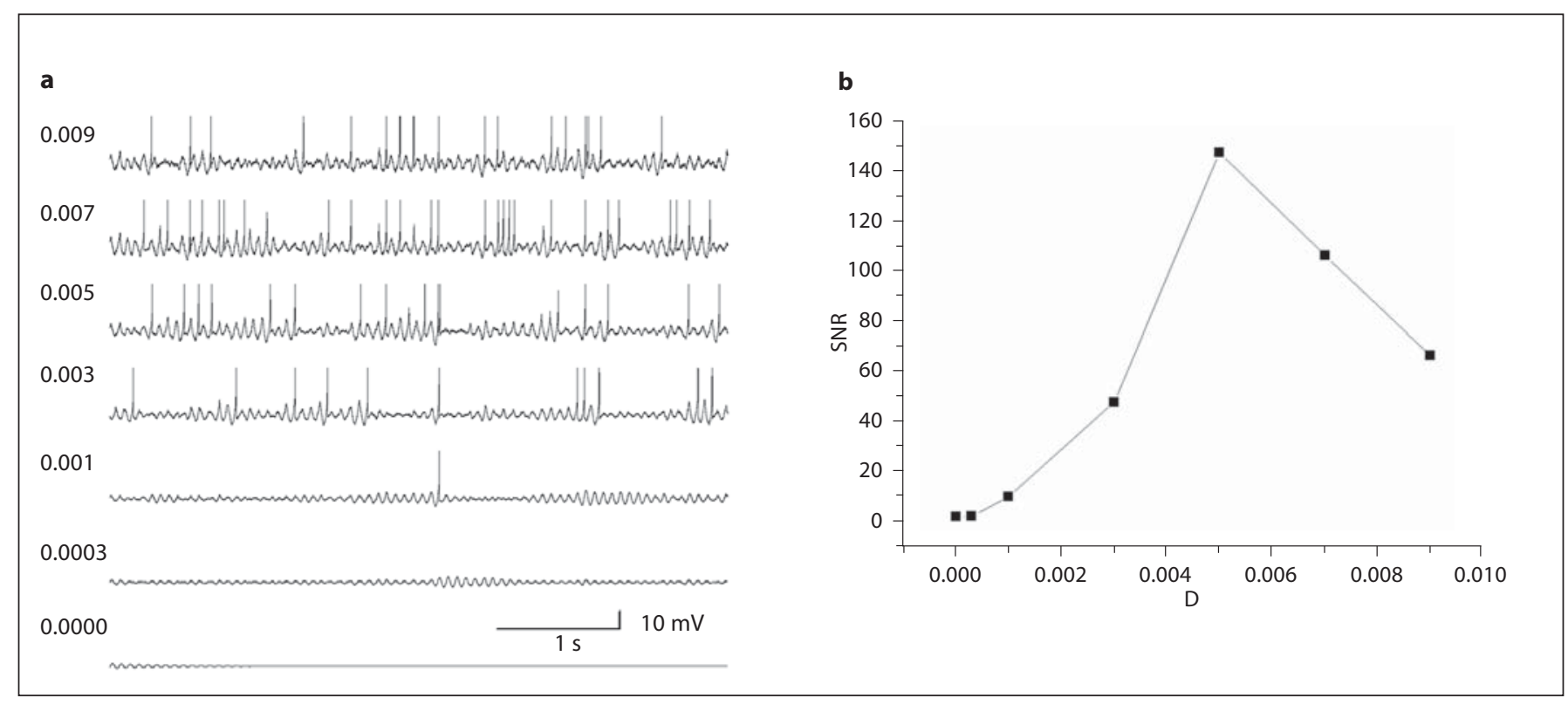

Fig. 5. Theoretical simulation for the effects of noise on electrical activities. a Membrane potentials at a fixed holding current $(1.3 \mathrm{nA})$ calculated through a mathematical model that includes the Hodgkin-Huxley currents $\left(I_{\mathrm{k}}\right.$ and $\left.I_{\mathrm{Na}}\right)$. Sustained oscillation emerged at small noise intensity of $0.0003 \mathrm{nA}$ r.m.s. and the am- plitude of oscillation increased with the increase of noise intensity. Intermittent spikes generated at noise intensity from 0.003 to 0.009 nA r.m.s. Scale (y, x) $10 \mathrm{mV}, 1,000 \mathrm{~ms}$. b SNR depends on noise intensity in the model behavior as shown in $\mathbf{a}$.

ment, when the $I_{\mathrm{Na}}$, P was blocked by a low-dose TTX, both subthreshold oscillations and discharges induced by noise were abolished completely (see fig. $4 \mathrm{~b}$ ). These results strongly suggest that both the stochastic flicker of channels and a certain intensity of $I_{\mathrm{Na}, \mathrm{P}}$ are the necessary conditions for noise-enhancing subthreshold oscillations in the injured DRG neurons. In addition, recent data from Huber and Braun [26] demonstrated that $I_{\mathrm{K}, \mathrm{S}}$ is an important factor for regulating the amplitude and frequency of oscillations. It is helpful to clarify the interaction of multiple channels in the subthreshold oscillations.

Nonlinear systems with noise can display SR-like behavior even without external periodic input. This phenomenon has been termed ASR $[4,12,13]$. Recently, ASR has attracted more interest in the mathematical neuron models $[13,27,28]$. It has been shown that the weak noise makes the membrane potential fluctuate near the firing threshold and displays sustaining subthreshold oscillation which is also called perithreshold oscillation. In the present study it was shown that ASR only appears obviously in the neurons with higher frequency resonance, suggesting that the intrinsic property of resonance is a decisive factor for occurring ASR behavior in neurons. This kind of neurons should belong to class 2 excitability 
which exhibits subthreshold oscillations before the occurrence of spikes and fires at a higher rate, though the rate is relatively independent of the strength of stimulation current [15, 16, 29-31]. In another word, at least in sensory neurons, only the neurons with class 2 excitability could exhibit ASR behavior. Increasing the noise intensity in $\mathrm{H}-\mathrm{H}$ neuronal model, the coherent motion, i.e. coherence between main frequency of electrical activity and intrinsic subthreshold oscillations, increases first to a maximum and then decreases, showing an optimal value of coherent motion to noise $[12,13,28]$. These theoretical studies suggest that the subthreshold oscillations which are determined by intrinsic resonance property in neurons are an essential condition for noise-inducing ASR behavior [19, 22, 32]. The present experimental data also showed that the threshold of first bursting firing decreased in the neurons with subthreshold oscillation as increasing noise intensity, in which SNR increased steeply at low noise levels and then fell gradually after peak value, suggesting a typical ASR appeared in this type of neurons. Meanwhile, a mathematical neuron model correctly predicted the behavior of real sensory neurons induced by noise. These experimental and mathematical results not only suggest that the intrinsic subthreshold oscillations in neurons represent an essential condition for noise-inducing ASR behavior but also provide direct evidence of ASR in the injured sensory neurons.

It is well known that the ectopic firing generated from the injured primary sensory neurons might contribute to chronic pain sensation $[15,20,21]$ and electrical mem- brane noise in neurons can alter excitability $[6,11]$. However, the relationship between the generation of chronic pain signal and intensity of noise is still ambiguous. The present work first suggests that electrical membrane noise may be a fundamental factor for promoting generation of chronic pain signals. Three lines of evidence support this possibility. First, $I_{\mathrm{Na}, \mathrm{P}}$ was evidenced as a main source of internal noise in neurons [6]. The present experiment further showed that in injured sensory neurons suppression of $I_{\mathrm{Na}, \mathrm{P}}$ can abolish the effects of noise. Second, the subthreshold membrane oscillation is an essential condition for generation of ectopic firings especially in some neurons with class 2 excitability, in which both membrane potentials for generating oscillation and amplitude of oscillation are dependent on the intensity of noise. Third, the formation of various ectopic firing patterns, for example, repetitive or bursting or irregular and so on, might be dependent on the interaction of subthreshold oscillation and noise through ASR behavior. In other words, after nerve injury, the neurons actually exist at a high level of noisy environment and possibly take advantage of or suffer from it.

\section{Acknowledgments}

We thank Prof. John Rinzel (Center for Neural Science and Courant Institute of Mathematical Sciences, New York University) for his critical reading and modification of the manuscript. This work was supported by National Scientific Foundation of China grants (30530260, 30870829, 30600581, 30930095).

\section{References}

-1 Braun HA, Wissing H, Schafer K, Hirsch MC: Oscillation and noise determine signal transduction in shark multimodal sensory cells. Nature 1994;367:270-273.

$>2$ Douglass JK, Wilkens L, Pantazelou E, Moss F: Noise enhancement of information transfer in crayfish mechanoreceptors by stochastic resonance. Nature 1993;365:337-340.

$>3$ Levin JE, Miller JP: Broadband neural encoding in the cricket cercal sensory system enhanced by stochastic resonance. Nature 1996;380:165-168.

4 Lindner B, Garcia-Ojalvo J, Neiman A, Schimansky-Geier L: Effects of noise in excitable systems. Phys Rep 2004;392:321-424.

$\checkmark 5$ Longtin A, Bulsara A, Moss F: Time-interval sequences in bistable system and the noiseinduced transmission of information by sensory neurons. Phys Rev Lett 1991;67:656659.
6 Dorval AD Jr, White JA: Channel noise is essential for perithreshold oscillations in entorhinal stellate neurons. J Neurosci 2005;25: 10025-10028.

7 Stacey WC, Durand DM: Synaptic noise improves detection of subthreshold signals in hippocampal CA1 neurons. J Neurophysiol 2001;86:1104-1112.

8 Reinker S, Puil E, Miura RM: Membrane resonance and stochastic resonance modulate firing patterns of thalamocortical neurons. J Comput Neurosci 2004;16:15-25.

$\checkmark 9$ Fernandez FR, White JA: Reduction of spike after depolarization by increased leak conductance alters interspike interval variability. J Neurosci 2009;29:973-986.

10 O'Gorman DE, White JA, Shera CA: Dynamical instability determines the effect of ongoing noise on neural firing. J Assoc Res Otolaryngol 2009;10:251-267.
White JA, Kay AR: Channel noise in neurons. Trends Neurosci 2000;23:131-137.

$12 \mathrm{Hu}$ G, Ditainger T, Ning CZ, Haken H: Stochastic resonance without external periodic force. Phys Rev Lett 1993;71:807-810.

13 Yu Y, Wang W, Wang J, Liu F: Resonanceenhanced signal detection and transduction in the Hodgkin-Huxley neuronal systems. Phys Rev E 2001;63:1-12.

14 Dong $\mathrm{H}$, Fan YH, Wang YY, Wang WT, Hu SJ: Lidocaine suppresses subthreshold oscillations by inhibiting persistent $\mathrm{Na}^{+}$current in injured dorsal root ganglion neurons. Physiol Res 2008;57:639-645.

15 Xie RG, Duan JH, Wang WT, Liu YH, Xing JL, Hu SJ: The classification of excitability and its ion channel mechanism in DRG neurons underlying chronic pain signal. Chin J Neuroanat 2010;26:268-272. 
16 Yang RH, Wang WT, Chen JY, Xie RG, Hu SJ: Gabapentin selectively reduces persistent sodium current in injured type-A dorsal root ganglion neurons. Pain 2009;143:48-55.

$\checkmark 17 \mathrm{Hu}$ SJ, Xing JL: An experimental model for chronic compression of dorsal root ganglion produced by intervertebral foramen stenosis in the rat. Pain 1998;77:15-23.

18 Azouz R, Gray CM: Cellular mechanisms contributing to response variability of cortical neurons in vivo. J Neurosci 1999;15: 2209-2223.

19 Gutfreund Y, Yarom YF, Segev I: Subthreshold oscillations and resonant frequency in guinea-pig cortical neurons: physiology and modeling. J Physiol 1995;483:621-640.

20 Amir R. Michaelis M, Devor M: Membrane potential oscillations in dorsal root ganglion neurons: role in normal electrogenesis and neuropathic pain. J Neurosci 1999;19:85898596.

-21 Xing JL, Hu SJ, Long KP: Subthreshold membrane potential oscillations of type A neurons in injured DRG. Brain Res 2001;901: $128-136$.
22 Hutcheon B, Yarom Y: Resonance, oscillation and the intrinsic frequency preferences of neurons. Trends Neurosci 2000;23:216222.

$23 \mathrm{Wu} \mathrm{N}$, Enomoto A, Tanaka S, Hsiao CF, Nykamp DQ, Izhikevich E, Chandler SH: Persistent sodium currents in mesencephalic $\mathrm{V}$ neurons participate in burst generation and control of membrane excitability. J Neurophysiol 2005;93:2710-2722.

24 Kovalsky Y, Amir R, Devor M: Subthreshold oscillations facilitate neuropathic spike discharge by overcoming membrane accommodation. Exp Neurol 2008;210:194-206.

25 Xie RG, Zheng DW, Xing JL, Zhang XJ, Song Y, Xie YB, Kuang F, Wang WT, Dong H, You SW, Xu H, Hu SJ: The blockade of persistent sodium currents contributed to the inhibition of riluzole on the spontaneous activity and subthreshold membrane potential oscillation in chronically compressed A-type dorsal root ganglion neurons. PLoS ONE, accepted.

26 Huber MT, Braun HA: Stimulus-response curves of a neuronal model for noisy subthreshold oscillations and related spike generation. Phys Rev E 2006;73:041929.
27 Gong PL, Xu JX, Hu SJ: Resonance in a noisedriven excitable neuron model. Chaos Solitons Fractals 2002;13:885-895.

28 Lee SG, Neiman A, Kim S: Coherence resonance in a Hodgkin-Huxley neuron. Phys Rev E 1998;57:3292-3297.

29 Liu YH, Yang J, Hu SJ: Transition between two excitabilities in mesencephalic $\mathrm{V}$ neurons. J Comput Neurosci 2008;24:95-104.

30 Yang J, Xing JL, Wu NP, Liu YH, Zhang CZ, Kuang F, Han VZ, HU SJ: Membrane current-based mechanisms for excitability transitions in neurons of the rat mesencephalic trigeminal nuclei. Neuroscience 2009;163: 799-810.

31 Izhikevich EM: Dynamical Systems in Neuroscience: The Geometry of Excitability and Bursting. Berlin, Springer, 2004, pp 195-204.

32 Braun HA, Voigt K, Huber MT: Oscillations, resonances and noise: basis of flexible neuronal pattern generation. Biosystems 2003;71: 39-50. 